\title{
Conics from symmetric Pythagorean triple preserving matrices
}

\author{
Mircea Crasmareanu* \\ (Communicated by Ion Mihai)
}

\begin{abstract}
The aim of this paper is to introduce and study the class of conics provided by symmetric Pythagorean triple preserving matrices. This class depends on three real parameters and various relationships between these parameters give special subclasses. A symmetric matrix of Barning and its associated hyperbola are carefully analyzed through a pair of points of rational coordinates. We transform and extend this latter hyperbola to a class of hyperbolas containing integral points $(k, k+3),(k+3, k)$. A complex approach is also included.
\end{abstract}

\section{Introduction}

Twenty years ago the problem of finding Pythagorean triple preserving matrices was studied in a couple of papers, namely [9] and [10]. Since then, this subject was also treated in some papers. For example, the present author gives in [4] a projective perspective on this topic while one of the authors of [9]-[10] returns to the subject in [12] and [13].

The starting point of this short note is the article [8] where very interesting relationships between Pythagorean triple preserving matrices (PTPM) and conics are pointed out. More precisely, we associate naturally conics to symmetric PTPMs and hence we produce a class of remarkable conics, called PTP-conics. We study this special class in both Euclidean coordinates $(x, y)$ as well as complex variable $z \in \mathbb{C}$ following the approach of [5]. Since symmetric PTPMs depend on three real parameters $(r, s, u)$ we discuss various types of PTP-conics imposing (algebraic) conditions on $(r, s, u)$, for example $r= \pm u$ and $r u=s^{2}$. The geometrical invariants of PTP-conics are computed as functions on $(r, s, u)$.

A PTP-hyperbola $\Gamma^{b}$ is associated to a symmetric PTMP matrix provided in 1963 by F. J. M. Barning. We find a pair of rational points on $\Gamma^{b}$ and hence a scaling in plane yields a hyperbola $\Gamma^{B}$ with a pair of integral points. The involved integers $0<n<m$ gives a Pythagorean triple $\left(m^{2}-n^{2}, 2 m n, m^{2}+n^{2}\right)$. We generalize this construction for points $(k, k+3),(k+3, k)$ with $k \in \mathbb{N}^{*}$ obtaining a new hyperbola depending on $k$ and called Barning type hyperbola with the associated Pythagorean triple $\left(6 k+9,2 k^{2}+6 k, 2 k^{2}+6 k+9\right)$.

Other remarkable examples of PTP conics are obtained when $r, s$ and $u$ form a geometric progression as well as they are exactly components of a Pythagorean triple. Also, the 2D Barning symmetric matrix yields some interesting objects, namely a binary quadratic form $F_{b}$ and an associated elliptic curve $E_{b}$, studied in the second section. Again, the Pell equation corresponding to a second binary quadratic form $F^{b}$ is connected with Heronian triangles and on this way we close a circular trajectory returning to the classical Pythagorean triple $(3,4,5)$. 


\section{Conics provided by symmetric PTPMs}

In the setting of two-dimensional Euclidean space $\left(\mathbb{R}^{2}, g_{c a n}=\operatorname{diag}(1,1)\right)$ let us consider the conic $\Gamma$ implicitly defined by $f \in C^{\infty}\left(\mathbb{R}^{2}\right)$ as: $\Gamma=\left\{(x, y) \in \mathbb{R}^{2} \mid f(x, y)=0\right\}$ where $f$ is a quadratic function of the form $f(x, y)=r_{11} x^{2}+2 r_{12} x y+r_{22} y^{2}+2 r_{10} x+2 r_{20} y+r_{00}$ with $r_{11}^{2}+r_{12}^{2}+r_{22}^{2} \neq 0$.

The study of $\Gamma$ is based on the symmetric matrices ( $e$ means extended):

$$
\Gamma:=\left(\begin{array}{ll}
r_{11} & r_{12} \\
r_{12} & r_{22}
\end{array}\right) \in \operatorname{Sym}(2), \quad \Gamma^{e}:=\left(\begin{array}{ccc}
r_{11} & r_{12} & r_{10} \\
r_{12} & r_{22} & r_{20} \\
r_{10} & r_{20} & r_{00}
\end{array}\right) \in \operatorname{Sym}(3) .
$$

For example, the algebraic invariants associated to $\Gamma$ :

$$
I:=r_{11}+r_{22}=\operatorname{Tr} \Gamma, \quad \delta:=\operatorname{det} \Gamma, \quad \Delta:=\operatorname{det} \Gamma^{e}, \quad D=: \delta+r_{11} r_{00}-r_{10}^{2}+r_{22} r_{00}-r_{20}^{2} .
$$

A matrix $X \in M_{3}(\mathbb{R})$ is called PTPM if maps any Pythagorean triple $(a<b<c) \in \mathbb{N}^{*}$ into another Pythagorean triple; sometimes is useful to point out that the rational point $\left(\frac{a}{c}, \frac{b}{c}\right)$ belongs to the positive quadrant of the unit circle $S^{1}$. Its expression is known from [9] or [4] to depend on 4 real numbers $r, t, s, u$ and in order to avoid ratios we write the double of $X$ as:

$$
2 X=2 X(r, t, s, u):=\left(\begin{array}{ccc}
\left(r^{2}-t^{2}\right)-\left(s^{2}-u^{2}\right) & 2(r s-t u) & \left(r^{2}-t^{2}\right)+\left(s^{2}-u^{2}\right) \\
2(r t-s u) & 2(r u+s t) & 2(r t+s u) \\
\left(r^{2}+t^{2}\right)-\left(s^{2}+u^{2}\right) & 2(r s+t u) & \left(r^{2}+t^{2}\right)+\left(s^{2}+u^{2}\right)
\end{array}\right) .
$$

The symmetrical condition is also known from [3] to be equivalent with $t=s$ :

$$
2 X=2 X(r, s, u):=\left(\begin{array}{ccc}
r^{2}-2 s^{2}+u^{2} & 2 s(r-u) & r^{2}-u^{2} \\
2 s(r-u) & 2\left(r u+s^{2}\right) & 2 s(r+u) \\
r^{2}-u^{2} & 2 s(r+u) & r^{2}+2 s^{2}+u^{2}
\end{array}\right)
$$

and a special set is studied in [1]. Hence, we arrive at our new geometrical objects:

Definition 1.1. A PTP-conic is a conic depending on $(r, s, u) \in \mathbb{R}^{3}$ in the form:

$$
\Gamma(r, s, u):\left(r^{2}-2 s^{2}+u^{2}\right) x^{2}+4 s(r-u) x y+2\left(r u+s^{2}\right) y^{2}+2\left(r^{2}-u^{2}\right) x+4 s(r+u) y+\left(r^{2}+2 s^{2}+u^{2}\right)=0 .
$$

A straightforward computation yields:

Proposition 1.1. The invariants of $\Gamma(r, s, u)$ are:

$$
\begin{gathered}
I(r, s, u)=(r+u)^{2} \geq 0, \quad \delta(r, s, u)=2\left(r u-s^{2}\right)\left(r^{2}+u^{2}+2 s^{2}\right), \\
D(r, s, u)=4\left(r u-s^{2}\right)\left(r^{2}+u^{2}+r u+s^{2}\right), \\
\Delta(r, s, u)=8\left(r u-s^{2}\right)^{3} .
\end{gathered}
$$

The PTP-conic $\Gamma$ is invariant to homogeneous scalings $(r, s, u) \rightarrow(\lambda r, \lambda s, \lambda u), \lambda \neq 0$.

Example 1.1. The vanishing $I=0$ is equivalent with $u=-r$. Hence we have the PTP-conic:

$$
\Gamma^{1}(r, s):\left(r^{2}-s^{2}\right)\left(x^{2}-y^{2}\right)+4 r s x y+\left(r^{2}+s^{2}\right)=0
$$

with the invariants:

$$
I^{1}=0, \quad \delta^{1}(r, s)=D(r, s)=-4\left(r^{2}+s^{2}\right)^{2}<0, \quad \Delta^{1}(r, s)=-8\left(r^{2}+s^{2}\right)^{3}<0
$$

and then $\Gamma^{1}$ is an equilateral hyperbola; for $r s \neq 0$ this hyperbola has as center the origin $O(0,0)$. Some examples are:

$$
\Gamma^{1}(r \neq 0, r): 2 x y+1=0, \quad \Gamma^{1}(r \neq 0,0): x^{2}-y^{2}+1=0, \quad \Gamma^{1}(0, s \neq 0): x^{2}-y^{2}-1=0 .
$$


Example 1.2. Returning to the general conic $\Gamma(r, s, u)$ the vanishing of the coefficient of $x y$ (and also of $x$ ) means $u=r$ and we get:

$$
\Gamma^{2}(r, s):\left(r^{2}-s^{2}\right) x^{2}+\left(r^{2}+s^{2}\right) y^{2}+4 r s y+\left(r^{2}+s^{2}\right)=0
$$

with the invariants:

$$
I^{2}(r, s)=4 r^{2} \geq 0, \delta^{2}(r, s)=4\left(r^{2}-s^{2}\right)\left(r^{2}+u^{2}\right), D^{2}(r, s)=4\left(r^{2}-s^{2}\right)\left(3 r^{2}+s^{2}\right), \Delta^{2}(r, s)=8\left(r^{2}-s^{2}\right)^{3} .
$$

For example:

$$
\Gamma^{2}(r \neq 0, r):(y+1)^{2}=0, \quad \Gamma^{2}(r \neq 0,0): x^{2}+y^{2}+1=0, \quad \Gamma^{2}(0, s \neq 0)=\Gamma^{1}(0, s)
$$

which means that $\Gamma^{2}(r, r)$ is the double line $y=-1, \Gamma^{2}(r, 0)$ is the empty conic while $\Gamma^{2}(0, s)$ is the equilateral hyperbola $x^{2}-y^{2}-1=0$.

Example 1.3. Recall that a primitive Pythagorean triple, or PPT on short, is a Pythagorean triple $(a, b, c)$ with $\operatorname{gcd}(a, b)=1$. Barning gave in [2] a systematic procedure to generate all PPTs with the help of iterated multiplication of the minimal (or Egypthian) Pythagorean triple $(3,4,5)$ (or $(4,3,5)$ ) with the following unimodular matrices:

$$
N_{1}=\left(\begin{array}{lll}
1 & -2 & 2 \\
2 & -1 & 2 \\
2 & -2 & 3
\end{array}\right), \quad N_{2}=\left(\begin{array}{lll}
1 & 2 & 2 \\
2 & 1 & 2 \\
2 & 2 & 3
\end{array}\right), \quad N_{3}=\left(\begin{array}{lll}
-1 & 2 & 2 \\
-2 & 1 & 2 \\
-2 & 2 & 3
\end{array}\right) .
$$

The Barning procedure is analyzed in [11] from a dynamical point of view. It follows only $N_{2}$ as symmetric PTPM sending $(3,4,5)$ to the Pythagorean triple $(21,20,29)$ and being:

$$
N_{2}=2 X\left(r=\sqrt{2}, s=\frac{1}{\sqrt{2}}, u=0\right) .
$$

Hence we have the Barning conic:

$$
\Gamma^{b}: x^{2}+4 x y+y^{2}+4 x+4 y+3=0
$$

containing the rational points $M_{1}\left(-\frac{1}{3},-\frac{4}{3}\right), M_{2}\left(-\frac{4}{3},-\frac{1}{3}\right)$ and having the center $C\left(-\frac{2}{3},-\frac{2}{3}\right)$. Since its invariants are:

$$
I^{b}=2, \quad \delta^{b}=-3, \quad D^{b}=-5, \quad \Delta^{b}=-1
$$

it follows that $\Gamma^{b}$ is a hyperbola with the canonical form:

$$
\Gamma^{b}: \frac{u^{2}}{-\frac{\Delta^{b}}{\delta^{b} \lambda_{1}}}+\frac{v^{2}}{-\frac{\Delta^{b}}{\delta^{b} \lambda_{2}}}-1=0
$$

with $\lambda_{1}>\lambda_{2}$ the eigenvalues of $\Gamma^{b} \in \operatorname{Sym}(2)$. Since $\lambda_{1}=3$ and $\lambda_{2}=-1$ it follows the canonical form of the Barning hyperbola:

$$
\Gamma^{b}: 9 u^{2}-3 v^{2}-1=0
$$

with the minor semiaxis $\frac{1}{3}$ and major semiaxis $\frac{1}{\sqrt{3}}$. In the canonical coordinates the above rational points are $M_{1}\left(-\frac{1}{3 \sqrt{2}},-\frac{1}{\sqrt{2}}\right), M_{2}\left(-\frac{1}{3 \sqrt{2}}, \frac{1}{\sqrt{2}}\right)$.

With the transform (a scaling one) $\left(x=-\frac{X}{3}, y=-\frac{Y}{3}\right)$ we obtain the hyperbola:

$$
\Gamma^{B}: X^{2}+4 X Y+Y^{2}-12(X+Y)+27=0
$$

with the integral points $M_{3}(1,4), M_{4}(4,1)$ and center $C(2,2)$. Recall that two positive integers $0<n<m$ yields a Pythagorean triple:

$$
a=m^{2}-n^{2}, \quad b=2 m n, \quad c=m^{2}+n^{2}
$$

and then to $(n=1, m=4)$ it corresponds the Pythagorean triple $(15,8,17)$ while the above $(21,20,29)$ corresponds to $(n=2, m=5)$. With a new transformation (a translation one) $(X=x-1, Y=y-1)$ in $(1.21)$ we have the Barning type hyperbola:

$$
\mathcal{H}^{b}: x^{2}+4 x y+y^{2}-18(x+y)+57=0
$$

having the integral points $M_{5}(2,5), M_{6}(5,2)$ and the center $C(3,3)$. We arrive at a new object: 
Definition 1.2. A Barning type matrix is a 3-symmetrical matrix depending on two integers:

$$
B\left(t_{1}, t_{2}\right)=\left(\begin{array}{ccc}
1 & 2 & t_{1} \\
2 & 1 & t_{1} \\
t_{1} & t_{1} & t_{2}
\end{array}\right) \in \operatorname{Sym}(3, \mathbb{Z})
$$

and its Barning type hyperbola is:

$$
\Gamma^{b}\left(t_{1}, t_{2}\right): x^{2}+4 x y+y^{2}+2 t_{1}(x+y)+t_{2}=0 .
$$

It follows, $\Gamma^{B}=\Gamma^{b}(-6,27)$ and $\mathcal{H}^{b}=\Gamma^{b}(-9,57)$. More generally, let $k \in \mathbb{N}^{*}$ and the transformation $(X=$ $x-(k-1), Y=y-(k-1))$ in (1.21). We get the Barning type hyperbola:

$$
\Gamma^{b}\left(-3(k+1), 3\left(2 k^{2}+4 k+3\right)\right): x^{2}+4 x y+y^{2}-6(k+1)(x+y)+3\left(2 k^{2}+4 k+3\right)=0
$$

having the integral points $M^{1}(k, k+3), M^{2}(k+3, k)$ and the center $C(k+1, k+1)$. The Pythagorean triple associated to $(k, k+3)$ is:

$$
a=6 k+9, \quad b=2 k^{2}+6 k, \quad c=2 k^{2}+6 k+9 .
$$

For $k=1$ we have the pair $\left(\Gamma^{B},(15,8,17)\right)$ with the points $\left(M_{3}, M_{4}\right)$ and for $k=2$ we have the pair $\left(\mathcal{H}^{b},(21,20,29)\right)$ with the points $\left(M_{5}, M_{6}\right)$.

The determinant of the Barning type matrix is:

$$
\operatorname{det} B\left(t_{1}, t_{2}\right)=2 t_{1}^{2}-3 t_{2}
$$

and the parabola given by $\operatorname{det} B(x, y)=-1$ has two families of integral points:

$$
P: 2 x^{2}-4 y=-1, \quad M_{7, n}\left(3 n+1,6 n^{2}+4 n+1\right), \quad M_{8, n}\left(3 n+2,6 n^{2}+8 n+3\right), \quad n \in \mathbb{N} .
$$

The parabola $P$ has the vertex $V\left(0, \frac{1}{3}\right)$ and the focus $F\left(0, \frac{17}{24}\right)$; its image together with the hyperbola $\Gamma^{b}$ is plotted below. The initial Barning matrix $N_{2}$ from (1.15) corresponds to $M_{8,0}$.

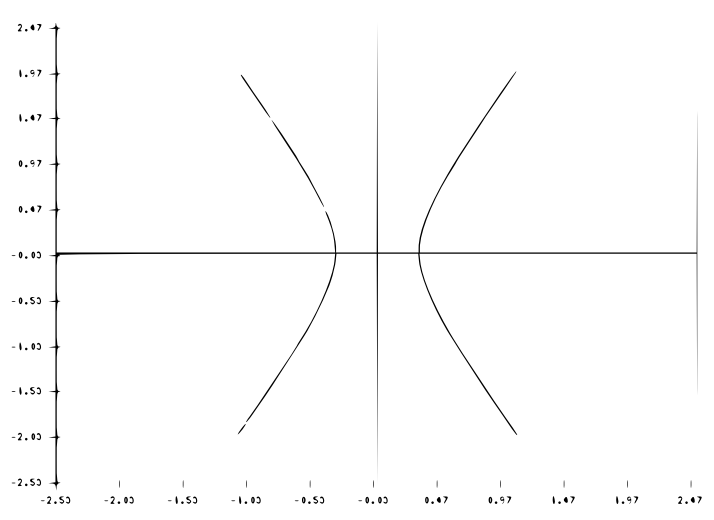

Figure 1. Hyperbola $\Gamma^{b}: 9 u^{2}-3 v^{2}-1=0$

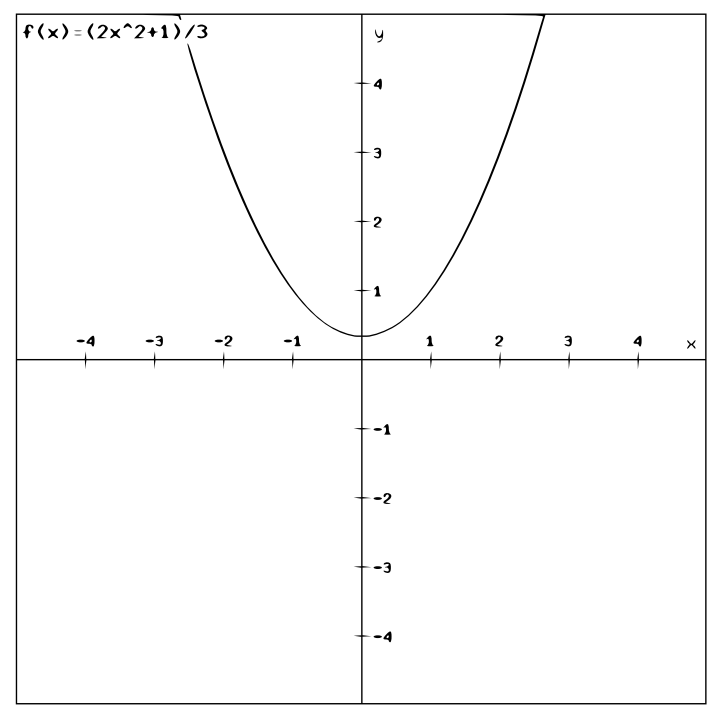

Figure 2. Parabola $P: 2 x^{2}-3 y=-1$

Example 1.4. Another remarkable class of PTP-conics is provided by the condition $r u=s^{2}$ since then:

$$
\delta=D=\Delta=0
$$

and the expression (1.5) of the conic is a perfect square:

$$
\Gamma\left(r u=s^{2}\right):[(r-u) x+2 s y+(r+u)]^{2}=0 .
$$


In fact, $r u=s^{2}$ means that $r, s$ and $u$ form a geometrical progression: $s=r q$ and $u=q^{2}$. It results the conic:

$$
\Gamma(q):\left[\left(1-q^{2}\right) x^{2}+2 q y+\left(1+q^{2}\right)\right]^{2}=0
$$

and we recognize the rational parametrization of $S^{1}$ :

$$
S^{1}: \cos \alpha=\frac{1-q^{2}}{1+q^{2}}, \quad \sin \alpha=\frac{2 q}{1+q^{2}}, \quad q=\tan \frac{\alpha}{2}
$$

giving a new expression of the conic:

$$
\Gamma(\alpha):[\cos \alpha \cdot x+\sin \alpha \cdot y+1]^{2}=0 .
$$

Example 1.5. Suppose that exactly the given parameters $r, s, u$ form a Pythagorean triple expressed through relations (1.22) as functions of $m, n \in \mathbb{N}^{*}$. We get the conic:

$$
\Gamma(m, n):\left(m^{4}-4 m^{2} n^{2}+n^{4}\right) x^{2}-8 m n^{3} x y+\left(n^{4}+4 m^{2} n^{2}-n^{4}\right) y^{2}-4 m^{2} n^{2} x+8 m^{3} n y+\left(m^{4}+4 m^{2} n^{2}+n^{4}\right)=0,
$$

with the invariants:

$$
\left\{\begin{array}{l}
I(m, n): 4 m^{4}>0, \quad \delta(m, n)=4\left[m^{8}-\left(n^{4}+4 m^{2} n^{2}\right)^{2}\right], \\
D(m, n)=4\left(m^{4}-n^{4}-4 m^{2} n^{2}\right)\left(3 m^{4}+3 n^{4}+4 m^{2} n^{2}\right), \Delta(m, n)=8\left(m^{4}-n^{4}-4 m^{2} n^{2}\right)^{3} .
\end{array}\right.
$$

For example, since the Pythagorean triple $(3,4,5)$ is generated by $(m=2, n=1)$ we associate:

$$
\Gamma(2,1): x^{2}-16 x y+31 y^{2}-16 x+64 y+33=0
$$

which is a hyperbola with center $C\left(\frac{8}{33},-\frac{32}{33}\right)$.

\section{A complex approach to PTP-conics}

The aim of this section is to study the PTP-conic $\Gamma$ by using the complex structure of the plane. More precisely, with the usual notation $z=x+i y \in \mathbb{C}$ we derive the complex expression of a general conic $\Gamma$ :

$$
\Gamma: F(z, \bar{z}):=A z^{2}+B z \bar{z}+\bar{A} \bar{z}^{2}+C z+\bar{C} \bar{z}+r_{00}=0
$$

with:

$$
A=\frac{r_{11}-r_{22}}{4}-\frac{r_{12}}{2} i \in \mathbb{C}, \quad 2 B=r_{11}+r_{22}=I \in \mathbb{R}, \quad C=r_{10}-r_{20} i \in \mathbb{C} .
$$

It follows that the usual rotation performed to eliminate the mixed term $x y$ has the meaning to reduce/rotate $A$ in the real line while the translation which eliminates the term $y$ has a similar meaning with respect to $C$. The inverse relationship between $f$ and $F$ is:

$$
r_{11}=B+2 \Re A, \quad r_{22}=B-2 \Re A, \quad r_{12}=-2 \Im A, \quad r_{10}=\Re C, \quad r_{20}=-\Im C
$$

with $\Re$ and $\Im$ respectively the real and imaginary part.

The linear invariant $I$ and the quadratic invariant $\delta$ are the trace respectively the determinant of the Hermitian matrix:

$$
\Gamma^{c}=\left(\begin{array}{cc}
B & 2 \bar{A} \\
2 A & B
\end{array}\right)
$$

which is a special one, the entries of the main diagonal being equal; hence their set is the three-dimensional subspace $\operatorname{Sym}(2)$ of the four-dimensional real linear space of $2 \times 2$ Hermitian matrices.

For our PTP-conic (1.5) we have the new coefficients:

$$
A(r, s, u)=\left(\frac{r-u}{2}-i s\right)^{2}, \quad B(r, s, u)=\left(\frac{r+u}{\sqrt{2}}\right)^{2}, \quad C(r, s, u)=\frac{r+u}{2}\left(\frac{r-u}{2}-i s\right)
$$

which are 2-homogeneous functions of $(r, s, u)$ and satisfy the quadratic relation:

$$
A B=2 C^{2} .
$$

Hence, a multiplication with $B$ of equation (2.1) gives a new relation for the PTP-conic, expressed only in $B$ and $C$ :

$$
\Gamma(r, s, u): 2(C z)^{2}+(B|z|)^{2}+2(\bar{C} \bar{z})^{2}+B\left(C z+\bar{C} \bar{z}+r_{00}(r, s, u)\right)=0 .
$$


Example 2.1. Both $A$ and $C$ from (2.5) are real if and only if $s=0$. We get the PTP-conic:

$$
\left\{\begin{array}{l}
\Gamma^{3}(r, u):\left(r^{2}+u^{2}\right) x^{2}+2 r u y^{2}+2\left(r^{2}-u^{2}\right) x+\left(r^{2}+u^{2}\right)=0 \\
A^{3}(r, u)=\left(\frac{r-u}{2}\right)^{2}, \quad C^{3}(r, u)=\frac{r^{2}-u^{2}}{4}
\end{array}\right.
$$

Its invariants are:

$$
I^{3}(r, u)=(r+u)^{2}, \delta^{3}(r, u)=2 r u\left(r^{2}+u^{2}\right), D^{3}(r, u)=4 r u\left(r^{2}+u^{2}+r u\right), \Delta^{3}(r, u)=8(r u)^{3} .
$$

Some examples are:

$$
\Gamma^{3}(r, r): x^{2}+y^{2}+1=0, \quad \Gamma^{3}(r, 0):(x+1)^{2}=0, \quad \Gamma^{3}(0, u):(x-1)^{2}=0 .
$$

The triple $(x, y, z)=\left(A^{3}, B^{3}, C^{3}\right)$ provides a rational parametrization of the cone:

$$
\mathcal{C}: x y=2 z^{2} .
$$

Example 2.2. Let us call the complex number:

$$
z_{\Gamma}:=\frac{r-u}{2}-i s \in \mathbb{C}
$$

as being affix of $\Gamma$, even in the case when $z_{\Gamma}$ does not belongs to $\Gamma$. For the example of the Barning hyperbola (1.17) we have:

$$
z_{\Gamma^{b}}=\frac{1-i}{\sqrt{2}}=e^{-\frac{i \pi}{4}} \notin \Gamma^{b}
$$

The rational points $M_{1}, M_{2} \in \Gamma^{b}$ have the affix:

$$
z_{1}=-\frac{1}{3}-\frac{4 i}{3}=-\frac{1}{3 \sqrt{17}}\left(\frac{1}{\sqrt{17}}+\frac{4}{\sqrt{17}} i\right), \quad z_{2}=-\frac{1}{3 \sqrt{17}}\left(\frac{4}{\sqrt{17}}+\frac{1}{\sqrt{17}} i\right)
$$

and the minimal polynomial of $\frac{1}{\sqrt{17}}+\frac{4}{\sqrt{17}} i \in S^{1}$ respectively $\frac{4}{\sqrt{17}}+\frac{1}{\sqrt{17}} i \in S^{1}$ is:

$$
p_{3}(x)=17 x^{4}+30 x^{2}+17, \quad p_{4}(x)=17 x^{4}-30 x^{2}+17 .
$$

The minimal polinomial of $\frac{2}{\sqrt{29}}+\frac{5}{\sqrt{29}} i \in S^{1}$ respectively $\frac{5}{\sqrt{29}}+\frac{2}{\sqrt{29}} i \in S^{1}$ :

$$
p_{5}(x)=29 x^{4}+42 x^{2}+29, \quad p_{6}(x)=29 x^{4}-42 x^{2}+29 .
$$

Example 2.3. In [6, p. 7-8] it is proved that the Barning matrix $N_{2}$ can be generated by a modified Farey tree and the matrix:

$$
A_{2}=\left(\begin{array}{cc}
0 & 1 \\
1 & 2
\end{array}\right)
$$

The associated binary quadratic form is:

$$
f(x, y)=f_{A_{2}}(x, y)=2 x y+2 y^{2}
$$

and its complex variant is:

$$
F_{A_{2}}(z, \bar{z})=\frac{i}{2}\left(\bar{z}^{2}-z^{2}\right)-\frac{1}{2}(\bar{z}-z)^{2}=-\frac{1+i}{2} z^{2}+z \bar{z}-\frac{1-i}{2} \bar{z}^{2}
$$

which means that $A=-\frac{1+i}{2}=-\frac{1}{\sqrt{2}} \overline{\bar{T}^{b}} \in \mathbb{C}$ and $B=1$. The equation (2.6) is:

$$
C^{2}=\frac{A B}{2}=-\frac{1+i}{4}=\frac{\sqrt{2}}{4}\left(-\frac{1}{\sqrt{2}}-\frac{i}{\sqrt{2}}\right)=2^{-\frac{3}{2}} e^{\frac{5 \pi}{4} i}
$$

with solutions:

$$
C_{ \pm}= \pm 2^{-\frac{3}{4}} e^{\frac{5 \pi}{8} i}
$$


Similar, the 2-block common of all Barning type hyperbolas is:

$$
\left(\begin{array}{ll}
1 & 2 \\
2 & 1
\end{array}\right)
$$

with associated coefficients $A=-i \in \mathbb{C}$ and $B=1$. Again the equation (2.6) is:

$$
C^{2}=\frac{A B}{2}=-\frac{i}{2}=\left(\frac{1-i}{2}\right)^{2}, \quad C_{ \pm}= \pm \frac{1-i}{2}= \pm \frac{1}{\sqrt{2}} z_{\Gamma^{b}} .
$$

Remark 2.1. Recall after [?] that the binary quadratic form:

$$
F(x, y)=a x^{2}+2 b x y+c y^{2}
$$

has the discriminant $\Delta(F):=4\left(b^{2}-a c\right)$ and is called indefinite if $\Delta(F)>0$. Also, an indefinite quadratic form is called reduced if:

$$
|\sqrt{\Delta(F)}-2| a||<2 b<\sqrt{\Delta(F)} .
$$

The Barning quadratic form given by (2.21):

$$
F_{b}(x, y)=x^{2}+4 x y+y^{2}, \quad \Delta\left(F_{b}\right)=12
$$

is indefinite but not reduced since the right inequality (2.23) is not satisfied. But for $\Delta=12$ with the formula (2) of [?, p. 4] we get another binary quadratic form, called principal:

$$
F^{b}(x, y):=x^{2}-\frac{\Delta}{4} y^{2}=x^{2}-3 y^{2} .
$$

If $x$ is a positive solution of the Pell equation $F^{b}(x, y)=1$ then $n-1, n=2 x, n+1$ are the edges of an Heronian triangle, when all the lengths of three edges of it as well as its area are integers; in fact it is a special Heronian triangle since its edge lengths are consecutive integers and [7] is a recent study in this type of triangles. The easy solution $(x=2, y=1)$ yields exactly the classical Pythagorean triple $(3,4,5)$ as edges on a special and right Heronian triangle.

Remark 2.2. In [?, p. 12] to every binary quadratic form $F$ is associated an elliptic curve:

$$
E_{F}: y^{2}=x^{3}+\left(2 b a^{-\frac{2}{3}}\right) x^{2}+\left(c a^{-\frac{1}{3}}\right) x, \quad \Delta\left(E_{F}\right)=16\left(\frac{c}{a}\right)^{2} \Delta(F) .
$$

Hence from $F_{b}$ of $(2.24)$ a Barning elliptic curve is obtained:

$$
E_{b}: y^{2}=x^{3}+4 x^{2}+x, \quad \Delta\left(E_{b}\right)=16 \cdot 12=2^{6} \cdot 3=192 .
$$

\section{Acknowledgments}

The author is indebted to two anonymous referees for their useful remarks.

\section{References}

[1] Austin, H. W. and Austin, J. W., On a special set of symmetric Pythagorean triple preserving matrices. Adv. Appl. Math. Sci. 12 (2012), no. 2, 97-104.

[2] Barning, F. J. M., On Pythagorean and quasi-Pythagorean triangles and a generation process with the help of unimodular matrices (Dutch) Math. Centrum, Amsterdam, Afd. Zuivere Wisk. ZW, 1963-011, 37 p.

[3] Bruening, J. T., Lohmeier, T. R. and Sebaugh, C. L., Symmetric Pythagorean triple preserving matrices. Missouri J. Math. Sci. 13 (2001), no. $1,4-16$.

[4] Crasmareanu, M., A new method to obtain Pythagorean triple preserving matrices. Missouri J. Math. Sci. 14 (2002), no. 3, 149-158.

[5] Crasmareanu, M., A complex approach to the gradient-type deformations of conics. Bull. Transilv. Univ. Braşov, Ser. III, Math. Inform. Phys. 10(59) (2017), no. 2, 59-62.

[6] Katayama, Shin-ichi, Modified Farey trees and Pythagorean triples. J. Math., Univ. Tokushima 47 (2013), 1-13.

[7] Murasaki, T., On the Heronian triple $(n+1, n, n-1)$ (Japanese). Sci. Rep. Fac. Educ. Gunma Univ. 52 (2004), 9-15. 
[8] Murru, N., Abrate, M., Barbero, S. and Cerruti, U., Groups and monoids of Pythagorean triples connected to conics. Open Math. 15 (2017), 1323-1331.

[9] Palmer, L., Ahuja, M. and Tikoo, M., Finding Pythagorean triple preserving matrices. Missouri J. Math. Sci. 10 (1998), no. 2, 99-105.

[10] Palmer, L., Ahuja, M. and Tikoo, M., Constructing Pythagorean triple preserving matrices. Missouri J. Math. Sci. 10 (1998), no. 3, $159-168$.

[11] Romik, D., The dynamics of Pythagorean triples. Trans. Am. Math. Soc. 360 (2008), no. 11, 6045-6064.

[12] Tikoo, M., A note on Pythagorean triple preserving matrices. Internat. J. Math. Ed. Sci. Tech. 33 (2002), no. 6, 893-894.

[13] Tikoo, M. and Wang, H., Generalized Pythagorean triples and Pythagorean triple preserving matrices. Missouri J. Math. Sci. 21 (2009), no. $1,3-12$.

MiRCEA CRASMAREANU

AdDRESS: Faculty of Mathematics, University "Al. I.Cuza", Iasi, 700506, România

E-MAIL: mcrasm@uaic.ro

ORCID ID: 0000-0002-5230-2751 